\title{
8-cyanobenzothiazinone analogs with potent antitubercular activity
}

\author{
Gang Zhang ${ }^{1} \cdot$ Li Sheng $^{1} \cdot$ Pooja Hegde ${ }^{2} \cdot$ Yan Li $^{1} \cdot$ Courtney C. Aldrich $\mathbb{D}^{1,2}$
}

Received: 10 November 2020 / Accepted: 1 December 2020 / Published online: 13 January 2021

(c) The Author(s), under exclusive licence to Springer Science+Business Media, LLC part of Springer Nature 2021

\begin{abstract}
8-Nitrobenzothiazinones (BTZs) exemplified by macozinone are a new class of antitubercular agents with exceptionally potent activity. The aryl nitro group has been considered indispensable for activity since this is bioactivated within mycobacteria by the flavoenzyme DprE1 to a reactive nitroso metabolite that covalently labels Cys387. However, the aryl nitro group is a potential liability with regards to safety, stability, and resistance. In this paper, we introduced a nitrile as a bioisosteric replacement of the nitro group, which we hypothesize can maintain a similar covalent mechanism of inhibition, but mitigate against the aforementioned concerns. 8-cyanobenzothiazinone 1d displayed potent antitubercular activity with an MIC of $130 \mathrm{nM}$ and had an improved volume of distribution in mice that increased the intrinsic half-life by twofold compared to macozinone. Analysis of the C-2 substituent of $\mathbf{1 d}$ revealed similar structure-activity relationships as observed for macozinone. Overall, the results confirm the 8-nitro group of benzothiazinones can be successfully replaced with a nitrile to retain useful activity and favorable pharmacokinetic properties.
\end{abstract}

\section{Graphical Abstract}

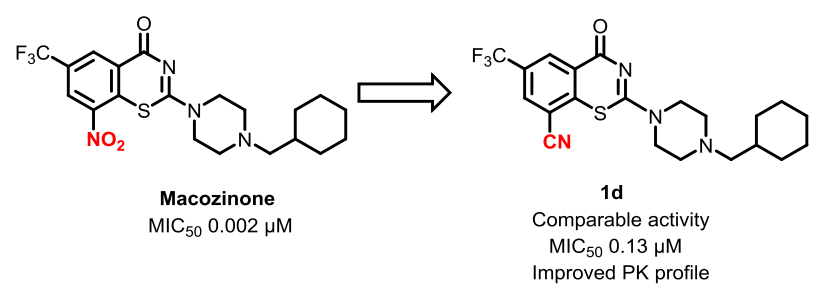

\section{Introduction}

Tuberculosis (TB) is one of the top 10 causes of death worldwide and the leading cause from a single infectious agent [1]. Moreover, nearly one quarter of the world's

Supplementary information The online version of this article (https:// doi.org/10.1007/s00044-020-02676-4) contains supplementary material, which is available to authorized users.

Courtney C. Aldrich

aldri015@umn.edu

1 State Key Laboratory of Bioactive Substances and Function of Natural Medicine, Institute of Materia Medica, Peking Union Medical College and Chinese Academy of Medical Sciences, Beijing 100050, China

2 Department of Medicinal Chemistry, University of Minnesota, Minneapolis, MN 55455, USA population are latently infected with Mycobacterium tuberculosis (Mtb). Reactivation of latent TB occurs when the immune system is weakened through aging, HIV coinfection, malnutrition, or diabetes. The Covid-19 pandemic and continued rise of drug-resistant TB threatens the modest gains made in recent years to bring TB back under control [1]. The development of new therapeutic agents, improved diagnostics, and effective vaccines will be required to achieve the WHO End TB strategy to decrease the TB incidence rate and the number of TB deaths by $80-90 \%$ within the next decade [1].

The benzothiazinones initially reported in 2009 have attracted consider attention for their potent antimycobacterial activity against drug-susceptible and resistant strains, synergy with other TB drugs, and outstanding in vivo activity in murine TB models [2-5]. Optimization of the initial lead candidate BTZ043 led to Macozinone (PBTZ169) with improved drug disposition properties and enhanced in vitro and in vivo activity 
Fig. 1 Mechanism of benzothiazinone inactivation of DprE1. a Mechanism of 8nitrobenzothiazinones. b Proposed covalent mechanism of 8-cyano benzothiazinones
A

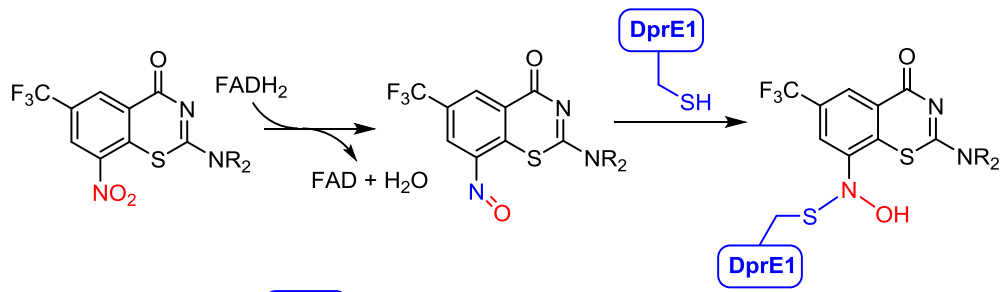

B

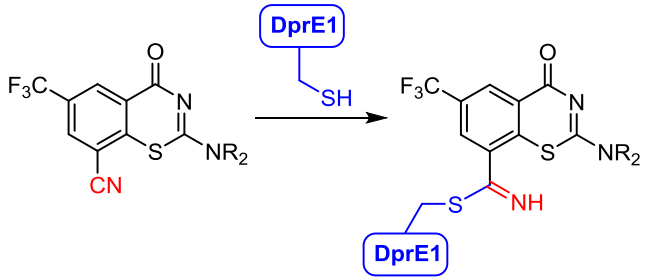

$[5,6]$. Macozinone successfully completed phase I clinical studies (NCT03036163 and NCT03423030); however phase 2a clinical trials to evaluate the early bactericidal activity were halted due to low enrollment (NCT03334734).

The benzothiazinones inhibit the biosynthesis of the arabinans, which form an integral part of the mycobacterial cell envelope [3, 4, 7-9]. The flavoenzyme DprE1 catalyzes the penultimate biosynthetic step of the decaprenylphospho-D-arabinose building block for construction of the highly branched arabinoglycan polymer, which links the inner peptidoglycan layer to the outer mycomembrane. Following binding to DprE1, the benzothiazinone 8-nitro group is reduced by the flavin cofactor of DprE1 to a nitroso metabolite, which covalently reacts with Cys387 of DprE1 to form a stable semimercaptal adduct (Fig. 1a) [10, 11]. The initially reported structure-activity relationships (SAR) demonstrated a requirement for a nitro group at C-8, a strongly electron-withdrawing group at C-6, no substituents at C-5 or C-7, a carbonyl at C-4 and sulfur at the first position [12-14]. The amino group at the $\mathrm{C}-2$ position is the most tolerant to modification, consequently this position has been the most extensively explored to modulate potency and drug disposition properties [3, 6, 15-33]. However, the 8 -nitro group remains a potential liability for idiosyncratic toxicity, metabolism by abundant commensal bacterial nitroreductases, reductive activation by glutathione and other biological thiolates and observed drug resistance by mutation of Cys387 to Ser387 [34-37].

Replacement of the C-8 nitro group of the benzothiazinones with pyrrole and triazole groups, which cannot covalently react with Cys387, afforded compounds with modest micromolar activity suggesting 'the invariant C-8 nitro group' may be more tolerant to modification than originally suggested $[12,17]$. We hypothesize that bioisosteric replacement of the 8-nitro group with a nitrile will maintain activity by enabling reaction with Cys 387 or the Cys387Ser mutant to form a covalent (thio)imidate adduct, but mitigate some of the undesirable features of the nitro

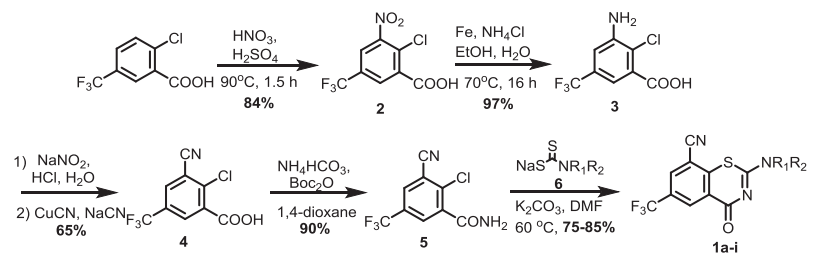

Scheme 1 Synthesis of 8-cyano-benzothiazinones 1a-i

group (Fig. 1b). The use of a nitrile to react with a nucleophilic serine or cysteine residue in an enzyme active site to form a reversible, but covalent, adduct has been demonstrated for Saxagliptin, a proline dipeptidase inhibitor used for type-2 diabetes and for Odanacatib, an investigator drug for osteoporosis that inhibits cathepsin K [38]. Qiao and co-workers recently disclosed the first successful bioisosteric replacement of the 8-nitro group with a nitrile [39]. Herein, we report our independent studies confirming this initial finding as well as the first SAR studies of 8-cyano derivatives.

Benzothiazinones containing an 8-cyano substituent were synthesized using an adaptation of the initial Makarov route starting from 2-chloro-5-(trifluoromethyl) benzoic acid, which was nitrated using concentrated sulfuric and nitric acid to provide known intermediate $\mathbf{2}$ (Scheme 1) [40]. Subsequent nitro reduction with iron powder in aqueous ethanol provided aniline $\mathbf{3}$ that was smoothly converted to aryl nitrile $\mathbf{4}$ through diazotization followed by Sandmeyer reaction with $\mathrm{CuCN}$. Formation of the resultant benzamide derivative $\mathbf{5}$ was accomplished under mild conditions employing di-tert-butyl dicarbonate and ammonium bicarbonate [41]. Condensation of benzamide 5 with a variety of sodium carbodithioates $\mathbf{6 a - i}$ in DMF through nucleophilic aromatic substitution followed by intramolecular cyclization and dehydrosulfidation yielded target compounds $1 \mathbf{1 a - i}$ in good yields without competitive reaction with the nitrile $[13,42]$. 


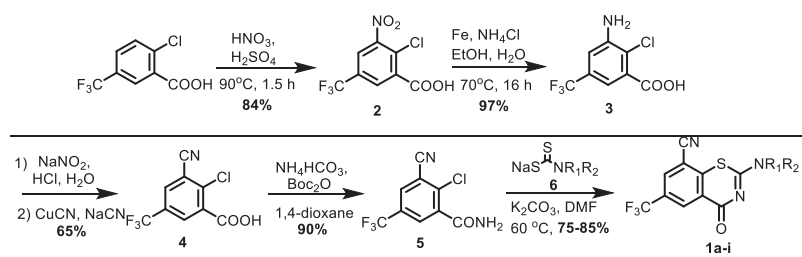

The 8-cyanobenzothiazinone derivatives and macozinone as a positive control were screened for antimycobacterial activity against $M t b \mathrm{H} 37 \mathrm{Rv}$ to determine the minimum inhibitory concentration (MIC) resulting in complete inhibition of observable mycobacterial growth (Table 1). Compound 1d containing the optimal cyclohexylmethylpiperazine substituent found in macozinone displayed an MIC of $130 \mathrm{nM}$ providing a rare example of a des-nitro benzothiazinone analog with potent nanomolar activity serving to validate our design principle. We next investigated the SAR of the cyclohexyl group with ring homologues 1a-c, but only cyclopentyl methyl 1c retained activity with an MIC of $200 \mathrm{nM}$ while 1a-b were inactive. While oxetanylmethyl 1f was inactive consistent with the data obtained from cyclobutylmethyl $\mathbf{1 b}$, we were pleasantly surprised to observe tetrahydrofuran-3-ylmethyl $\mathbf{1 e}$ incorporating a polar oxygen atom retained moderate activity showing only a 10 -fold loss of potency relative to cyclopentyl methyl 1c. Modification of the piperazine by methylation in $\mathbf{1 g}-\mathbf{h}$ or replacement with the bicyclic isostere in 1i abolished activity. Taken together, the limited SAR of this nitrile series demonstrates the cyclohexylmethylpiperazine substituent is optimal, only very conservative modifications are tolerated in the cycloalkyl substituent, and the piperazine must be strictly maintained. The active compound 1c, $\mathbf{1 d}$, and $\mathbf{1 e}$ were further evaluated for potential cytotoxicity against Vero cells, but none demonstrated any inhibition of cellular growth at the highest concentration evaluated $\left(\mathrm{CC}_{50}>150 \mu \mathrm{M}\right)$ furnishing a therapeutic index $\left(\mathrm{MIC}_{\mathrm{CC}} \mathrm{C}_{50}\right)$ of greater than 1000 for $\mathbf{1 d}$.

The calculated and experimentally determined physicochemical properties of the analogs and macozinone are shown in Tables 2 for comparison. We calculated the overall lipophilicity as measured by the partition coefficient $\operatorname{cog} \mathrm{P}$, the lipophilic ligand efficiency (LLE, $\log$ MIC $-\log \mathrm{P})$, the total polar surface area (tPSA) and experimentally measured the melting points as a proxy for compound solubility [43]. Replacement of the C-8 nitro group of macozinone with a nitrile in 1d results in a modest decrease in $\operatorname{cog} \mathrm{P}$ of only 0.3 units. However, the LLE for 1d decreases by 1.5 units relative to macozinone, due to the loss of antimycobacterial activity. The nitrile analog does significantly reduce the tPSA, which is expected to enhance membrane permeability. Among the other active nitrile analogs, compound 1e containing a tetrahydrofuran-3-ylmethyl substituent had an improved LLE attributed to the dramatically lower clogP that offset the loss of potency.

The metabolic stability of 1d and macozinone were evaluated using mouse and human liver microsomes with and without NADPH. Macozinone was included as a positive control while samples without NADPH served as negative controls. In the absence of NADPH neither macozinone nor 1d were metabolized. The metabolic stability of macozinone was consistent with previous reports showing a half-life of 34-43 min [30]. Compound 1d was metabolized approximately twice as quickly in human liver microsomes, but nearly to the same extent in mouse liver microsomes compared to macozinone (Table 3 ).

Compound 1d and macozinone were evaluated in male ICR mice to determine their pharmacokinetic parameters. Compound 1d reached a maximum plasma concentration of $110 \mathrm{ng} / \mathrm{mL}$ or $240 \mathrm{nM}$, following oral dosing at $10 \mathrm{mg} /$ $\mathrm{mL}$ and showed a 2.1-fold increase in the intrinsic halflife relative to macozinone (determined from intravenously (i.v.) dosing). The increased half-life of $\mathbf{1 d}$ was driven by a corresponding 2.0-fold increase in the volume of distribution (V) since the clearance $(\mathrm{Cl})$ remained nearly unchanged. The enhanced volume of distribution may in turn reflect the reduced tPSA that can enhance membrane permeability. However, the oral bioavailability of $\mathbf{1 d}$ was decreased twofold relative to macozinone that may in turn potentially reflect lower solubility of 1d, which is predicted based on the substantially higher melting point of $\mathbf{1 d}$ compared to macozinone. While the increased volume of distribution is favorable, compounds 1d was deemed to possess inadequate PK parameters due to its low oral bioavailability. To the best of our knowledge, this report also provides the first data for the oral bioavailability of macozinone (Table 4).

In conclusion, we successfully replaced the 8-nitro group of macozinone with an isosteric nitrile moiety to address potential concerns of safety, stability, and resistance. Compound 1d, containing an identical C-2 substituent as found in macozinone, possessed respectable antimycobacterial activity with an MIC of $130 \mathrm{nM}$, displayed no cytotoxicity, and an improved intrinsic half-life owing to its greater volume of distribution. However, 1d has approximately twofold lower oral bioavailability than macozinone, which we demonstrated was also low. We explored the SAR of the C-2 substituent of $\mathbf{1 d}$ and showed only conservative modifications were tolerated. Further optimization at $\mathrm{C}-2$ will be required to enhance oral bioavailability and exposure as well as potency before the 8-cyano benzothiazinones can be advanced. 
Table 1 MIC and $\mathrm{CC}_{50}$ of compound $1 \mathrm{a}-\mathrm{i}$<smiles>[R]c1nc(=O)c2cc(C(F)(F)F)cc([N+](=O)[O-])c2s1</smiles><smiles>[R]c1nc(=O)c2cc(C(F)(F)F)cc(C#N)c2s1</smiles>

Compounds

${ }^{a}$ Minimum inhibitory concentration against $M$. tuberculosis H37Rv

${ }^{\mathrm{b}}$ The concentration required to decrease cell viability of Vero cells by $50 \%$; cnd $=$ not determined

\section{Methods}

\section{General}

Unless otherwise noted, reagents and materials were obtained from commercial suppliers and were used without further purification. Solvents were dried by the appropriate drying agents prior to use. Anhydrous tetrahydrofuran and dichloromethane were obtained from commercial sources.
Table 2 The ClogP, LLE, and melting point of $1 \mathrm{a}-\mathrm{i}$

\begin{tabular}{lllll}
\hline Compounds & $\mathrm{Clog} \mathrm{P}^{\mathrm{a}}$ & $\mathrm{LLE}^{\mathrm{b}}$ & $\mathrm{Mp}\left({ }^{\circ} \mathrm{C}\right)$ & tPSA \\
\hline Macozinone & 5.1 & 3.6 & $185-187$ & 87.7 \\
1a & 3.1 & - & $151-153$ & 59.7 \\
1b & 3.7 & - & $162-165$ & 59.7 \\
1c & 4.2 & 2.5 & $196-198$ & 59.7 \\
1d & 4.8 & 2.1 & $218-221$ & 59.7 \\
$1 \mathrm{e}$ & 2.0 & 3.8 & $173-175$ & 68.9 \\
1f & 1.3 & - & $172-174$ & 68.9 \\
1g & 5.8 & - & $182-185$ & 59.7 \\
1h & 5.8 & - & $180-182$ & 59.7 \\
1i & 4.1 & - & $193-195$ & 68.5 \\
\hline
\end{tabular}

${ }^{\mathrm{a}} \mathrm{C} \log \mathrm{P}$ and tPSA were determined by Chemdraw Professional Version 16.0

${ }^{\mathrm{b}}$ Lipophilic Ligand Efficiency (LLE) was calculated from the equation: $\mathrm{LLE}=\log _{10} \mathrm{MIC}-\mathrm{Clog}_{10} \mathrm{P}$

All solvents used for routine isolation of products and for chromatography were reagent grade. Moisture- and airsensitive reactions were carried out under an atmosphere of Argon. All reactions were monitored by thin layer chromatography (TLC) and column chromatography purification was performed using 230-400 mesh silica gel. NMR spectra were measured on Bruker AV400 spectrometer at 400 or $300 \mathrm{MHz}$ for $1 \mathrm{H}$ spectra and at 100 or $75 \mathrm{MHz}$ for ${ }^{13} \mathrm{C}$ spectra using $\mathrm{CDCl}_{3}, \mathrm{CD}_{3} \mathrm{OD}$, and $\mathrm{D}_{2} \mathrm{O}$, and calibrated from the residual solvent signal. All final products were characterized by ${ }^{1} \mathrm{H} \mathrm{NMR},{ }^{13} \mathrm{C} \mathrm{NMR}$, and MS analyses. Except for the known compounds, all new compounds were also characterized and confirmed by HRMS.

\section{2-chloro-3-nitro-5-(trifluoromethyl)benzoic acid (2)}

To a mixture of conc. $\mathrm{H}_{2} \mathrm{SO}_{4}(3.0 \mathrm{~mL})$ and conc. $\mathrm{HNO}_{3}$ (3.0 mL) was added 2-chloro-5-(trifluoromethyl)benzoic acid $(0.52 \mathrm{~g}, 2.32 \mathrm{mmol})$. After stirred for $1.5 \mathrm{~h}$ at $90{ }^{\circ} \mathrm{C}$, the reaction was cooled to room temperature and poured into ice water $(10 \mathrm{~mL})$, filtered and washed with cold water $(5.0 \mathrm{~mL})$, dried in vacuo to afford the title compound $(0.52 \mathrm{~g}, 84 \%)$ as a white solid: ${ }^{1} \mathrm{H}$ NMR (400 MHz, DMSO$\left.\mathrm{d}_{6}\right) \delta 8.72(\mathrm{~s}, 1 \mathrm{H}), 8.41(\mathrm{~s}, 1 \mathrm{H}) ;{ }^{13} \mathrm{C} \mathrm{NMR}\left(\mathrm{DMSO}-\mathrm{d}_{6}\right) \delta$ 164.8, 150.1, 136.1, $130.2(\mathrm{~d}, J=3.8 \mathrm{~Hz}), 129.4,129.0$, 127.7, and 124.4.

3-amino-2-chloro-5-(trifluoromethyl)benzoic acid (3)

A mixture of intermediate $2(0.5 \mathrm{~g}, 1.9 \mathrm{mmol}, 1.0$ equiv. $)$, reductive iron powder $(0.5 \mathrm{~g}, 8.7 \mathrm{mmol}, 4.6$ equiv. $), \mathrm{NH}_{4} \mathrm{Cl}$ ( $0.7 \mathrm{~g}, 14 \mathrm{mmol}, 7.4$ equiv.) in $60 \mathrm{~mL}$ of ethanol aqueous solution (ethanol: water, 5:1) was stirred at $70{ }^{\circ} \mathrm{C}$ and monitored by TLC. After $16 \mathrm{~h}$, the solvent was removed, and the 
Table 3 Microsomal stability of $\mathbf{1 d}$

\begin{tabular}{lllll}
\hline Compounds & \multicolumn{2}{l}{ Mouse } & \multicolumn{2}{l}{ Human } \\
\cline { 2 - 5 } & \multicolumn{2}{l}{ (\% remaining at $30 \mathrm{~min})$} & & \\
\cline { 2 - 5 } & With NADPH & Without NADPH & With NADPH & Without NADPH \\
\hline Macozinone & 50.7 & 102 & 46.8 & 101 \\
1d & 38.6 & 96.4 & 26.3 & 102 \\
\hline
\end{tabular}

Table 4 Pharmacokinetic parameters of macozinone and 1d in male ICR mice following oral or intravenous administration

\begin{tabular}{|c|c|c|c|c|c|}
\hline \multirow[t]{2}{*}{ Parameters } & \multirow[t]{2}{*}{ Units } & \multicolumn{2}{|c|}{ Macozinone } & \multicolumn{2}{|l|}{$1 d$} \\
\hline & & $\mathrm{PO}$ & iv & $\mathrm{PO}$ & iv \\
\hline $\mathrm{t} 1 / 2 \beta$ & $\mathrm{h}$ & 1.68 & 0.86 & 1.74 & 1.84 \\
\hline Tmax & $\mathrm{h}$ & 0.25 & 0.033 & 0.25 & 0.033 \\
\hline Cmax & $\mathrm{ng} / \mathrm{ml}$ & 251 & 1230 & 110 & 1335 \\
\hline $\operatorname{AUC}(0-t)$ & $\mathrm{h}^{\mathrm{a}} \mathrm{ng} / \mathrm{ml}$ & 354 & 423 & 174 & 445 \\
\hline $\mathrm{Vd}$ & $\mathrm{L} / \mathrm{kg}$ & - & 5.64 & - & 11.3 \\
\hline $\mathrm{Cl}$ & $\mathrm{ml} / \mathrm{min} / \mathrm{kg}$ & - & 75.8 & - & 71.2 \\
\hline $\mathrm{F}$ & $\%$ & 16.7 & - & 7.8 & - \\
\hline
\end{tabular}

${ }^{\mathrm{a}}$ The doses of oral and intravenous administration were 10 and $2 \mathrm{mg} / \mathrm{kg}$, respectively

residue was suspended in $10 \mathrm{~mL}$ of ethyl acetate. The suspension was filtered, and the filtrate was washed with $1 \mathrm{~N} \mathrm{HCl}$ $(10 \mathrm{~mL})$ and brine $(15 \mathrm{~mL})$, dried over $\mathrm{Na}_{2} \mathrm{SO}_{4}$, filtered and concentrated in vacuo to afford the title compound $(0.40 \mathrm{~g}$, 91\%) as a brown solid: $R_{\mathrm{f}}=0.36$ (DCM:MeOH, 8:1 with a trace amount of acetic acid); ${ }^{1} \mathrm{H}$ NMR $\left(400 \mathrm{MHz}\right.$, DMSO- $\left.\mathrm{d}_{6}\right)$ $\delta 13.53(\mathrm{~s}, 1 \mathrm{H}), 7.21(\mathrm{~s}, 1 \mathrm{H}), 7.09(\mathrm{~s}, 1 \mathrm{H}), 6.09(\mathrm{~s}, 2 \mathrm{H}) ;{ }^{13} \mathrm{C}$ NMR (DMSO-d $\left.{ }_{6}\right) \delta 166.9,147.0,134.2,128.3(\mathrm{~d}, J=$ $32.4 \mathrm{~Hz}), 125.5,122.8,118.4$, and $112.8(\mathrm{~d}, J=20.2 \mathrm{~Hz})$.

\section{2-chloro-3-cyano-5-(trifluoromethyl)benzoic acid (4)}

To a mixture of intermediate $3(0.63 \mathrm{~g}, 2.63 \mathrm{mmol}, 1.0$ equiv.) in $\mathrm{H}_{2} \mathrm{O}(5.0 \mathrm{~mL})$ and conc. $\mathrm{HCl}(0.4 \mathrm{~mL})$, was added dropwise a solution of $\mathrm{NaNO}_{2}(0.24 \mathrm{~g}, 3.42 \mathrm{mmol}, 1.3$ equiv.) in $\mathrm{H}_{2} \mathrm{O}(0.5 \mathrm{~mL})$ at $0{ }^{\circ} \mathrm{C}$. After stirred at $0-10{ }^{\circ} \mathrm{C}$ for $2 \mathrm{~h}$, the solution was added to a mixture of $\mathrm{CuCN}(0.23 \mathrm{~g}$, $2.63 \mathrm{mmol}, 1.0$ equiv.) and $\mathrm{NaCN}(0.19 \mathrm{~g}, 3.95 \mathrm{mmol}, 1.5$ equiv.) in $\mathrm{H}_{2} \mathrm{O}(3.0 \mathrm{~mL})$ at $0{ }^{\circ} \mathrm{C}$. The mixture was stirred at $70{ }^{\circ} \mathrm{C}$ for $6 \mathrm{~h}$ and cooled to room temperature. The $\mathrm{pH}$ value was adjusted to 1 with conc. $\mathrm{HCl}$. The crude product was extracted with ethyl acetate $(3 \times 10 \mathrm{~mL})$, washed with $1 \mathrm{~N}$ $\mathrm{HCl}(1 \mathrm{~mL})$, brine $(10 \mathrm{~mL})$, dried over $\mathrm{Na}_{2} \mathrm{SO}_{4}$, filtered and concentrated in vacuo. The crude product was purified by silica gel column chromatography (eluting with 100: 1 DCM: $\mathrm{MeOH})$ to afford the title compound $(0.43 \mathrm{~g}, 65 \%)$ as a colorless solid: $R_{\mathrm{f}}=0.47$ (DCM:MeOH, 8:1 with a trace amount of acetic acid). ${ }^{1} \mathrm{H}$ NMR $\left(400 \mathrm{MHz}, \mathrm{CD}_{3} \mathrm{OD}\right) \delta 8.37$ (s, 1H), 8.35 (s, 1H); ${ }^{13} \mathrm{C}$ NMR (151 MHz, CD3OD) $\delta$ 164.3, $139.1,133.8,133.2(\mathrm{~d}, J=3.9 \mathrm{~Hz}), 131.5(\mathrm{~d}, J=3.7 \mathrm{~Hz})$, $129.8,129.5,116.4$, and 114.0.

\section{2-chloro-3-cyano-5-(trifluoromethyl)benzamide (5)}

To a stirred solution of 2-chloro-3-cyano-5-(trifluoromethyl)benzoic acid $(0.25 \mathrm{~g}, 1.0 \mathrm{mmol}, 1.0$ equiv. $)$, pyridine $(0.05 \mathrm{~mL}$, $0.62 \mathrm{mmol}, 0.62$ equiv.), and $\mathrm{Boc}_{2} \mathrm{O}(0.28 \mathrm{~g}, 1.3 \mathrm{mmol}, 1.3$ equiv.) in 1,4-dioxane $(2.0 \mathrm{~mL})$ at room temperature was added ammonium bicarbonate $(0.10 \mathrm{~g}, 1.26 \mathrm{mmol}, 1.26$ equiv.). The reaction was stirred overnight at room temperature and then partitioned between EtOAc $(10.0 \mathrm{~mL})$ and $\mathrm{H} 2 \mathrm{O}$ $(10.0 \mathrm{~mL})$. The organic layer was separated, washed consecutively with water $(10.0 \mathrm{~mL})$ and $0.6 \mathrm{~N}$ aqueous $\mathrm{HCl}$ $(10.0 \mathrm{~mL})$, dried over anhydrous $\mathrm{Mg}_{2} \mathrm{SO}_{4}$, and filtered. The filtrate was concentrated under reduced pressure to afford the title compound $(0.22 \mathrm{~g}, 90 \%)$ as an off-white solid: ${ }^{1} \mathrm{H}$ NMR $\left(600 \mathrm{MHz}, \mathrm{CD}_{3} \mathrm{OD}\right) \delta 8.30(\mathrm{~d}, J=1.0 \mathrm{~Hz}, 1 \mathrm{H}), 8.09$ (d, $J=$ $1.0 \mathrm{~Hz}, 1 \mathrm{H}) ;{ }^{13} \mathrm{C}$ NMR $\left(151 \mathrm{MHz}, \mathrm{CD}_{3} \mathrm{OD}\right) \delta 168.8,138.3$, 133.2 (q, $J=3.6 \mathrm{~Hz}), 131.3(\mathrm{q}, J=34.4 \mathrm{~Hz}), 130.7$ (q, $J=$ $4.0 \mathrm{~Hz}), 124.0\left(\mathrm{q},{ }^{1} J_{\mathrm{C}-\mathrm{F}}=272.5 \mathrm{~Hz}\right), 116.8$, and 115.4 .

\section{General procedure for the synthesis of compounds 6a-i}

To a pre-cooled mixture of amine (2.04 mmol, 1 equiv) in EtOAc was added $30 \%$ aqueous $\mathrm{NaOH} \quad(0.68 \mathrm{~mL}$, 
$5.09 \mathrm{mmol}, 2.5$ equiv) followed by $\mathrm{CS}_{2} \quad(0.15 \mathrm{~mL}$, $2.45 \mathrm{mmol}, 1.2$ equiv). The reaction mixture was stirred for $3 \mathrm{~h}$ at $0{ }^{\circ} \mathrm{C}$, then at room temperature for another $3 \mathrm{~h}$. The reaction mixture was filtered and the filter cake was washed by ethyl acetate and dried under vacuum to afford intermediates 6a-i as published in our previous papers [30].

\section{General procedure for the synthesis of compounds 1a-i}

A mixture of intermediate $5(0.16 \mathrm{~g}, 0.61 \mathrm{mmol}, 1.2$ equiv), 6a-i ( $0.51 \mathrm{mmol}, 1.0$ equiv) and anhydrous $\mathrm{K} 2 \mathrm{CO} 3(0.08 \mathrm{~g}, 0.56 \mathrm{mmol}, 1.1$ equiv) was stirred in anhydrous DMF $(5 \mathrm{~mL})$ at $60^{\circ} \mathrm{C}$. After $2 \mathrm{~h}$, the reaction mixture was cooled down to room temperature and poured into ice water. The product was extracted with $\mathrm{CH}_{2} \mathrm{Cl}_{2}$ $(5 \times 20 \mathrm{~mL})$. The organic layer was combined, washed by saturated brine $(3 \times 5 \mathrm{~mL})$ and dried by anhydrous sodium sulfate which was removed by filtration after $20 \mathrm{~min}$. The solvent $\mathrm{CH}_{2} \mathrm{Cl}_{2}$ was removed under reduced pressure. The product was purified by silica flash column chromatography with the indicated solvent system (petroleum ether/

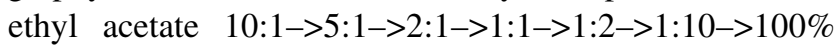
ethyl acetate).<smiles>N#Cc1cc(C(F)(F)F)cc2c(=O)nc(N3CCN(CC4CC4)CC3)sc12</smiles>

\section{2-(4-(cyclopropylmethyl)piperazin-1-yl)-4-oxo-6- (trifluoromethyl)-4H-benzo[e][1,3]thiazine-8- carbonitrile (1a)}

The title compound was prepared from sodium 4-(cyclopropylmethyl)piperazine-1-carbodithioate $\quad 6 a \quad(0.08 \mathrm{~g}$, $0.34 \mathrm{mmol}, \quad 1.0$ equiv) and 2-chloro-3-cyano-5-(trifluoromethyl)benzamide 5 ( $0.10 \mathrm{~g}, 0.41 \mathrm{mmol}, 1.2$ equiv) using the general procedure for cyclization to afford compound 1a $(0.12 \mathrm{~g}, 86 \%)$ as a light yellow solid: $\mathrm{mp}$ $151-153{ }^{\circ} \mathrm{C} ; \quad R_{\mathrm{f}}=0.20$ (1:2 Hexane-EtOAc); ${ }^{1} \mathrm{H}$ NMR $\left(300 \mathrm{MHz}, \mathrm{CDCl}_{3}\right) \delta 8.91(\mathrm{~d}, J=2.0 \mathrm{~Hz}, 1 \mathrm{H}), 8.08(\mathrm{~d}, J=$ $2.0 \mathrm{~Hz}, 1 \mathrm{H}), 4.19$ (br s, 2H), 3.83 (br s, 2H), 2.69 (br s, 4H), $2.34(\mathrm{~d}, J=6.6 \mathrm{~Hz}, 2 \mathrm{H}), 0.91-0.86(\mathrm{~m}, 1 \mathrm{H}), 0.60-0.54(\mathrm{~m}$, $2 \mathrm{H}), 0.16-0.11(\mathrm{~m}, 2 \mathrm{H}) ;{ }^{13} \mathrm{C}$ NMR $\left(75 \mathrm{MHz}, \mathrm{CDCl}_{3}\right) \delta$ $166.4,159.5,140.6,132.9$ (q, $J=2.6 \mathrm{~Hz}), 131.6$ (q, $J=$ $2.6 \mathrm{~Hz}), 130.9(\mathrm{q}, J=25.9 \mathrm{~Hz}), 124.8,122.5\left(\mathrm{q},{ }^{1} J_{\mathrm{C}-\mathrm{F}}=\right.$ $203.7 \mathrm{~Hz}), 114.0,111.1,63.3,52.5,47.0-46.3$ (m), 29.8, 8.2, and 4.1; HRMS (ESI+) $\mathrm{m} / \mathrm{z}[\mathrm{M}+\mathrm{H}]^{+}$calcd for $\mathrm{C}_{18} \mathrm{H}_{18} \mathrm{~F}_{3} \mathrm{~N}_{4} \mathrm{OS}$ : 395.1148; found 395.1144 (error $1.0 \mathrm{ppm}$ ).<smiles>N#Cc1cc(C(F)(F)F)cc2c(=O)nc(N3CCN(CC4CCC4)CC3)sc12</smiles>

\section{2-(4-(cyclobutylmethyl)piperazin-1-yl)-4-oxo-6- (trifluoromethyl)-4H-benzo[e][1,3]thiazine-8- carbonitrile (1b)}

The title compound was prepared from sodium 4-(cyclobutylmethyl)piperazine-1-carbodithioate $\mathbf{6 b}(0.09 \mathrm{~g}, 0.34 \mathrm{mmol}$, 1.0 equiv) and 2-chloro-3-cyano-5-(trifluoromethyl)benzamide $5(0.10 \mathrm{~g}, 0.41 \mathrm{mmol}, 1.2$ equiv) using the general procedure for cyclization to afford compound $\mathbf{1 b}(0.12 \mathrm{~g}$, $85 \%)$ as light yellow solid: $\mathrm{mp} 162-165^{\circ} \mathrm{C} ; R_{\mathrm{f}}=0.20(1: 2$ Hexane-EtOAc); ${ }^{1} \mathrm{H}$ NMR $\left(300 \mathrm{MHz}, \mathrm{CDCl}_{3}\right) \delta 8.91$ (d, $J=$ $2.0 \mathrm{~Hz}, 1 \mathrm{H}), 8.08(\mathrm{~d}, J=2.0 \mathrm{~Hz}, 1 \mathrm{H}), 4.13(\mathrm{~m}, 2 \mathrm{H}), 3.77(\mathrm{~m}$, $2 \mathrm{H}), 2.55-2.46(\mathrm{~m}, 7 \mathrm{H}), 2.10-2.04(\mathrm{~m}, 2 \mathrm{H}), 1.98-1.82(\mathrm{~m}$, 2H), $1.75-1.67(\mathrm{~m}, 2 \mathrm{H}) ;{ }^{13} \mathrm{C}$ NMR $\left(75 \mathrm{MHz}, \mathrm{CDCl}_{3}\right) \delta$ $166.2,159.2,140.5,132.7$ (q, $J=3.6 \mathrm{~Hz}), 131.4$ (q, $J=$ $3.6 \mathrm{~Hz}), 130.5(\mathrm{q}, J=34.7 \mathrm{~Hz}), 124.6,122.4\left(\mathrm{q},{ }^{1} J_{\mathrm{C}-\mathrm{F}}=\right.$ $271.4 \mathrm{~Hz}), 113.9,110.9,64.5,52.6,47.0-46.4(\mathrm{~m}), 33.4$, 27.6, and 18.8; HRMS (ESI+) $\mathrm{m} / \mathrm{z}[\mathrm{M}+\mathrm{H}]^{+}$calcd for $\mathrm{C}_{19} \mathrm{H}_{20} \mathrm{~F}_{3} \mathrm{~N}_{4} \mathrm{OS}$ : 409.1304; found 409.1302 (error $0.6 \mathrm{ppm}$ ).<smiles>N#Cc1cc(C(F)(F)F)cc2c(=O)nc(N3CCN(CC4CCCC4)CC3)sc12</smiles>

\section{2-(4-(cyclopentylmethyl)piperazin-1-yl)-4-oxo-6- (trifluoromethyl)-4H-benzo[e][1,3]thiazine-8- carbonitrile (1c)}

The title compound was prepared from sodium 4-(cyclopentylmethyl)piperazine-1-carbodithioate $\quad \mathbf{6 c} \quad(0.09 \mathrm{~g}$, $0.34 \mathrm{mmol}, \quad 1.0$ equiv) and 2-chloro-3-cyano-5-(trifluoromethyl)benzamide 5 ( $0.10 \mathrm{~g}, 0.41 \mathrm{mmol}, 1.2$ equiv) using the general procedure for cyclization to afford compound 1c $(0.12 \mathrm{~g}, 86 \%)$ as light yellow solid: $\mathrm{mp}$ $196-198^{\circ} \mathrm{C} ; R_{\mathrm{f}}=0.20$ (1:2 Hexane-EtOAc); ${ }^{1} \mathrm{H}$ NMR $\left(300 \mathrm{MHz}, \mathrm{CDCl}_{3}\right) \delta 8.91(\mathrm{~d}, J=2.0 \mathrm{~Hz}, 1 \mathrm{H}), 8.08(\mathrm{~d}, J=$ $2.0 \mathrm{~Hz}, 1 \mathrm{H}), 4.15(\mathrm{~m}, 2 \mathrm{H}), 3.78(\mathrm{~m}, 2 \mathrm{H}), 2.58(\mathrm{~m}, 4 \mathrm{H})$, 2.33-2.30 (m, 2H), 2.13-2.03 (m, 1H), 1.77-1.75 (m, 2H), $1.61-1.55(\mathrm{~m}, 4 \mathrm{H})$, and $1.24-1.18(\mathrm{~m}, 2 \mathrm{H}) ;{ }^{13} \mathrm{C} \mathrm{NMR}$ $\left(75 \mathrm{MHz}, \mathrm{CDCl}_{3}\right) \delta 166.3,159.3,140.6,132.7(\mathrm{q}, J=$ $3.6 \mathrm{~Hz}), 131.4(\mathrm{q}, J=3.6 \mathrm{~Hz}), 130.6(\mathrm{q}, J=34.7 \mathrm{~Hz})$, 
124.5, $122.4\left(\mathrm{q},{ }^{1} J_{\mathrm{C}-\mathrm{F}}=271.4 \mathrm{~Hz}\right), 113.9,110.9,63.9$, 52.7, 47.1-46.5 (m), 36.9, and 31.1, 25.1; HRMS (ESI+) $\mathrm{m} / \mathrm{z}[\mathrm{M}+\mathrm{H}]^{+}$calcd for $\mathrm{C}_{20} \mathrm{H}_{22} \mathrm{~F}_{3} \mathrm{~N}_{4} \mathrm{OS}$ : 423.1461; found 423.1457 (error $0.9 \mathrm{ppm}$ ).<smiles>N#Cc1cc(C(F)(F)F)cc2c(=O)nc(N3CCN(CC4CCCCC4)CC3)sc12</smiles>

\section{2-(4-(cyclohexylmethyl)piperazin-1-yl)-4-oxo-6- (trifluoromethyl)-4H-benzo[e][1,3]thiazine-8- carbonitrile (1d)}

The title compound was prepared from sodium 4-(cyclohexylmethyl)piperazine-1-carbodithioate $\mathbf{6 d}(0.10 \mathrm{~g}, 0.34 \mathrm{mmol}$, 1.0 equiv) and 2-chloro-3-cyano-5-(trifluoromethyl)benzamide 5 ( $0.10 \mathrm{~g}, 0.41 \mathrm{mmol}, 1.2$ equiv) using the general procedure for cyclization to afford compound $1 d(0.13 \mathrm{~g}$, $89 \%)$ as light yellow solid: mp $218-221^{\circ} \mathrm{C} ; R_{\mathrm{f}}=0.20(1: 2$ Hexane-EtOAc); ${ }^{1} \mathrm{H}$ NMR (400 MHz, $\left.\mathrm{CDCl}_{3}\right) \delta 8.91(\mathrm{~d}, J=$ $2.0 \mathrm{~Hz}, 1 \mathrm{H}), 8.07$ (d, J=2.0 Hz, 1H), 4.14 (s, 2H), 3.77 (s, $2 \mathrm{H}), 2.53(\mathrm{~s}, 4 \mathrm{H}), 2.18(\mathrm{~d}, J=7.1 \mathrm{~Hz}, 2 \mathrm{H}), 1.80-1.66(\mathrm{~m}$, $5 \mathrm{H}), 1.52-1.46(\mathrm{~m}, 1 \mathrm{H}), 1.29-1.15(\mathrm{~m}, 3 \mathrm{H}), 0.93-0.84(\mathrm{~m}$, $2 \mathrm{H}) ;{ }^{13} \mathrm{C}$ NMR $\left(101 \mathrm{MHz}, \mathrm{CDCl}_{3}\right) \delta 166.5,159.4,140.7$, $132.9(\mathrm{q}, J=3.7 \mathrm{~Hz}), 131.6(\mathrm{q}, J=3.6 \mathrm{~Hz}), 130.8(\mathrm{q}, J=$ $34.9 \mathrm{~Hz}), 124.8,122.5\left(\mathrm{q},{ }^{1} J_{\mathrm{C}-\mathrm{F}}=274.2 \mathrm{~Hz}\right), 114.1,111.1$, 65.2, 53.1, 47.2-46.7 (m), 35.1, 31.8, and 26.8, 26.1; HRMS $(\mathrm{ESI}+) \mathrm{m} / \mathrm{z}[\mathrm{M}+\mathrm{H}]^{+}$calcd for $\mathrm{C}_{21} \mathrm{H}_{24} \mathrm{~F}_{3} \mathrm{~N}_{4} \mathrm{OS}$ : 437.1617; found 437.1613 (error $1.0 \mathrm{ppm}$ ).<smiles>N#Cc1cc(C(F)(F)F)cc2c(=O)nc(N3CCN(CC4CCOC4)CC3)sc12</smiles>

\section{4-0xo-2-(4-((tetrahydrofuran-3-yl)methyl)piperazin- 1-yl)-6-(trifluoromethyl)-4H-benzo[e][1,3]thiazine-8- carbonitrile (1e)}

The title compound was prepared from sodium 4-((tetrahydrofuran-3-yl)methyl)piperazine-1-carbodithioate 6 $(0.09 \mathrm{~g}, 0.34 \mathrm{mmol}, 1.0$ equiv) and 2-chloro-3-cyano-5(trifluoromethyl)benzamide $5 \quad(0.10 \mathrm{~g}, \quad 0.41 \mathrm{mmol}, \quad 1.2$ equiv) using the general procedure for cyclization to afford compound 1e $(0.11 \mathrm{~g}, 76 \%)$ as light yellow solid: $\mathrm{mp}$ $173-175^{\circ} \mathrm{C} ; \quad R_{\mathrm{f}}=0.20 \quad\left(1: 2\right.$ Hexane-EtOAc); ${ }^{1} \mathrm{H}$ NMR $\left(300 \mathrm{MHz}, \mathrm{CDCl}_{3}\right): \delta 8.92(\mathrm{~d}, J=2.0 \mathrm{~Hz}, 1 \mathrm{H}), 8.08$ $(\mathrm{d}, J=2.0 \mathrm{~Hz}, 1 \mathrm{H}), 4.16-4.11(\mathrm{~m}, 2 \mathrm{H}), 3.91-3.72(\mathrm{~m}, 5 \mathrm{H})$, 3.59-3.54 (m, 1H), $2.62(\mathrm{~m}, 4 \mathrm{H}), 2.50-2.43(\mathrm{~m}, 2 \mathrm{H})$, 2.10-1.99 (m, 1H), 1.68-1.58 (m, 2H); ${ }^{13} \mathrm{C}$ NMR $(75 \mathrm{MHz}$, $\left.\mathrm{CDCl}_{3}\right) \delta 166.4,159.5,140.6,132.9(\mathrm{q}, J=2.7 \mathrm{~Hz}), 131.6$ $(\mathrm{q}, J=2.7 \mathrm{~Hz}), 130.9(\mathrm{q}, J=26.0 \mathrm{~Hz}), 124.8,122.5\left(\mathrm{q},{ }^{1} J_{\mathrm{C}-}\right.$ $\mathrm{F}=203.5 \mathrm{~Hz}), \quad 114.0, \quad 111.1, \quad 72.1, \quad 67.8, \quad 61.5, \quad 52.8$, 47.0-46.6 (m), 36.7, 30.6, and 29.8; HRMS (ESI+) m/z [M $+\mathrm{H}]^{+}$calcd for $\mathrm{C}_{19} \mathrm{H}_{20} \mathrm{~F}_{3} \mathrm{~N}_{4} \mathrm{O}_{2} \mathrm{~S}$ : 425.1254; found 425.1249 (error $1.1 \mathrm{ppm}$ ).<smiles>N#Cc1cc(C(F)(F)F)cc2c(=O)nc(N3CCN(CC4COC4)CC3)sc12</smiles>

\section{2-(4-(oxetan-3-ylmethyl)piperazin-1-yl)-4-oxo-6- (trifluoromethyl)-4H-benzo[e][1,3]thiazine-8- carbonitrile (1f)}

The title compound was prepared from sodium 4-(oxetan-3ylmethyl)piperazine-1-carbodithioate $6 \mathbf{f}(0.09 \mathrm{~g}, 0.34 \mathrm{mmol}$, 1.0 equiv) and 2-chloro-3-cyano-5-(trifluoromethyl)benzamide 5 ( $0.10 \mathrm{~g}, 0.41 \mathrm{mmol}, 1.2$ equiv) using the general procedure for cyclization to afford compound 1f $(0.10 \mathrm{~g}$, $72 \%)$ as light yellow solid: $\mathrm{mp} 172-174^{\circ} \mathrm{C} ; R_{\mathrm{f}}=0.20(1: 2$ Hexane-EtOAc); ${ }^{1} \mathrm{H} \mathrm{NMR}\left(300 \mathrm{MHz}, \mathrm{CDCl}_{3}\right) \delta 8.92(\mathrm{~d}$, $J=2.0 \mathrm{~Hz}, 1 \mathrm{H}), 8.08(\mathrm{~d}, J=2.0 \mathrm{~Hz}, 1 \mathrm{H}), 4.85-4.81(\mathrm{~m}$, 2H), 4.43-4.39 (m, 2H), 4.13 (m, 2H), 3.78 (m, 2H), 3.24 $(\mathrm{m}, 1 \mathrm{H}), 2.80-2.77(\mathrm{~m}, 2 \mathrm{H}), 2.56(\mathrm{~m}, 3 \mathrm{H})$, and $1.57(\mathrm{~m}$, $1 \mathrm{H}) ;{ }^{13} \mathrm{C} \mathrm{NMR}\left(75 \mathrm{MHz}, \mathrm{CDCl}_{3}\right) \delta 166.4,159.5,140.5$, $132.9(\mathrm{q}, J=2.6 \mathrm{~Hz}), 131.7(\mathrm{q}, J=2.7 \mathrm{~Hz}), 130.9$ (q, $J=$ $26.2 \mathrm{~Hz}), 124.7,122.5\left(\mathrm{q},{ }^{1} J_{\mathrm{C}-\mathrm{F}}=203.7 \mathrm{~Hz}\right), 114.0,111.1$, 61.5, 52.7, 46.5, 32.9, and 29.8; HRMS (ESI+) m/z [M + $\mathrm{H}]^{+}$calcd for $\mathrm{C}_{18} \mathrm{H}_{18} \mathrm{~F}_{3} \mathrm{~N}_{4} \mathrm{O}_{2} \mathrm{~S}$ : 411.1097; found 411.1094 (error $0.8 \mathrm{ppm}$ ).<smiles>C[C@H]1CN(CC2CCCCC2)C[C@@H](C)N1c1nc(=O)c2cc(C(F)(F)F)cc(C#N)c2s1</smiles>

\section{2-((2S,6R)-4-(cyclohexylmethyl)-2,6- dimethylpiperazin-1-yl)-4-oxo-6-(trifluoromethyl)- 4H-benzo[e][1,3]thiazine-8-carbonitrile (1g)}

The title compound was prepared from sodium $(2 S, 6 R)-4$ (cyclohexylmethyl)-2,6-dimethylpiperazine-1-carbodithioate $\quad \mathbf{6 i} \quad(0.10 \mathrm{~g}, \quad 0.34 \mathrm{mmol}, \quad 1.0 \quad$ equiv $)$ and 
2-chloro-3-cyano-5-(trifluoromethyl)benzamide $5(0.10 \mathrm{~g}$, $0.41 \mathrm{mmol}, 1.2$ equiv) using the general procedure for cyclization to afford compound $\mathbf{1 g}(0.13 \mathrm{~g}, 83 \%)$ as light yellow solid: $\mathrm{mp} 182-185^{\circ} \mathrm{C} ; R_{\mathrm{f}}=0.20$ (1:2 HexaneEtOAc); ${ }^{1} \mathrm{H}$ NMR $\left(300 \mathrm{MHz}, \mathrm{CDCl}_{3}\right) \delta 8.93(\mathrm{~d}, J=2.0 \mathrm{~Hz}$, $1 \mathrm{H}), 8.07(\mathrm{~d}, J=2.0 \mathrm{~Hz}, 1 \mathrm{H}), 5.21(\mathrm{~m}, 1 \mathrm{H}), 4.21(\mathrm{~m}, 1 \mathrm{H})$, 2.80-2.74 (m, 2H), 2.31-2.15 (m, 4H), 1.83-1.67 (m, 5H), $1.59-1.44(\mathrm{~m}, 7 \mathrm{H}), 1.32-1.11(\mathrm{~m}, 3 \mathrm{H}), 0.98-0.85$ (m, 2H); ${ }^{13} \mathrm{C}$ NMR $\left(75 \mathrm{MHz}, \mathrm{CDCl}_{3}\right) \delta 166.1,159.1,141.1,132.7$ $(\mathrm{q}, J=3.6 \mathrm{~Hz}), 131.5(\mathrm{q}, J=3.6 \mathrm{~Hz}), 130.5(\mathrm{q}, J=$ $34.6 \mathrm{~Hz}), 124.8,122.5$ (q, $J=271.4 \mathrm{~Hz}), 114.1,110.8$, $64.9,58.3,57.8,51.4,50.8,35.4,31.6,26.8,26.1,20.5$, and 20.1; HRMS (ESI+) $\mathrm{m} / \mathrm{z} \quad[\mathrm{M}+\mathrm{H}]^{+}$calcd for $\mathrm{C}_{23} \mathrm{H}_{28} \mathrm{~F}_{3} \mathrm{~N}_{4} \mathrm{OS}$ : 465.1930; found 465.1928 (error $0.5 \mathrm{ppm}$ ).

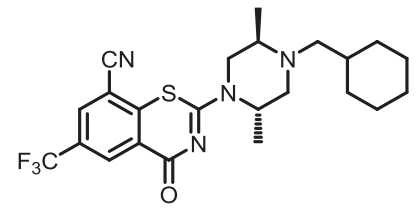

\section{2-((2S,5R)-4-(cyclohexylmethyl)-2,5- dimethylpiperazin-1-yl)-4-oxo-6-(trifluoromethyl)- $4 \mathrm{H}$-benzo[e][1,3]thiazine-8-carbonitrile $(1 \mathrm{~h})$}

The title compound was prepared from sodium $(2 S, 5 R)-4$ (cyclohexylmethyl)-2,5-dimethylpiperazine-1-carbodithioate 6m $(0.10 \mathrm{~g}, 0.34 \mathrm{mmol}, 1.0$ equiv) and 2-chloro-3-cyano-5(trifluoromethyl)benzamide 5 ( $0.10 \mathrm{~g}, 0.41 \mathrm{mmol}, 1.2$ equiv) using the general procedure for cyclization to afford compound $\mathbf{l h}(0.13 \mathrm{~g}, 80 \%)$ as light yellow solid: $\mathrm{mp} 180-182{ }^{\circ} \mathrm{C}$; $R_{\mathrm{f}}=0.20$ (1:2 Hexane-EtOAc); ${ }^{1} \mathrm{H}$ NMR $\left(300 \mathrm{MHz}, \mathrm{CDCl}_{3}\right)$ $\delta 8.92(\mathrm{~d}, J=2.0 \mathrm{~Hz}, 1 \mathrm{H}), 8.07(\mathrm{~d}, J=2.0 \mathrm{~Hz}, 1 \mathrm{H}), 5.35-3.11$ (m, 3H), $3.11(\mathrm{~m}, 1 \mathrm{H}), 2.84-2.80(\mathrm{~m}, 1 \mathrm{H}), 2.44-2.40(\mathrm{~m}, 1 \mathrm{H})$, 2.21-2.16 (m, 2H), 1.87-1.83 (m, 1H), 1.75-1.71 (m, 4H), $1.57-1.36(\mathrm{~m}, 4 \mathrm{H}), 1.21-1.14(\mathrm{~m}, 3 \mathrm{H}), 0.99-0.82(\mathrm{~m}, 5 \mathrm{H})$; ${ }^{13} \mathrm{C} \mathrm{NMR}\left(75 \mathrm{MHz}, \mathrm{CDCl}_{3}\right) \delta 166.4,160.0,140.7,132.8(\mathrm{q}, J$ $=3.6 \mathrm{~Hz}), 131.5(\mathrm{q}, J=3.6 \mathrm{~Hz}), 130.6(\mathrm{q}, J=34.6 \mathrm{~Hz})$, $124.8,122.4(\mathrm{q}, J=271.4 \mathrm{~Hz}), 114.0,110.9,61.1,53.2,50.8$, 49.3, 47.5, 35.3, 31.6, 31.6, 26.8, 26.1, 26.0, 16.4, and 7.7; HRMS (ESI+) $\mathrm{m} / \mathrm{z}[\mathrm{M}+\mathrm{H}]^{+}$calcd for $\mathrm{C}_{23} \mathrm{H}_{28} \mathrm{~F}_{3} \mathrm{~N}_{4} \mathrm{OS}$ : 465.1930; found 465.1930 (error $0.1 \mathrm{ppm}$ ).

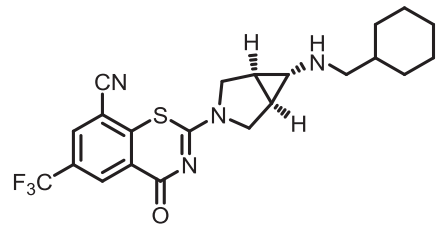

2-((1R,5S,6s)-6-((cyclohexylmethyl)amino)-3azabicyclo[3.1.0]hexan-3-yl)-4-oxo-6(trifluoromethyl)-4H-benzo[e][1,3]thiazine-8carbonitrile (1i)

The title compound was prepared from sodium $(1 R, 5 S, 6 S)$ 6-((cyclohexylmethyl)amino)-3-azabicyclo[3.1.0]hexane-3carbodithioate 6p $(0.10 \mathrm{~g}, 0.34 \mathrm{mmol}, 1.0$ equiv) and 2chloro-3-cyano-5-(trifluoromethyl)benzamide 5 (0.10 g, $0.41 \mathrm{mmol}, 1.2$ equiv) using the general procedure for cyclization to afford compound $1 \mathbf{i}(0.11 \mathrm{~g}, 71 \%)$ as light yellow solid: $\mathrm{mp} 193-195{ }^{\circ} \mathrm{C} ; R_{\mathrm{f}}=0.20 \quad(1: 2$ HexaneEtOAc); ${ }^{1} \mathrm{H}$ NMR $\left(300 \mathrm{MHz}, \mathrm{CDCl}_{3}\right) \delta 8.77(\mathrm{~d}, J=2.1 \mathrm{~Hz}$, $1 \mathrm{H}), 8.63(\mathrm{~d}, J=2.1 \mathrm{~Hz}, 1 \mathrm{H}), 3.95-3.91(\mathrm{~m}, 1 \mathrm{H}), 3.84-3.72$ $(\mathrm{m}, 3 \mathrm{H}), 3.34(\mathrm{~m}, 2 \mathrm{H}), 2.04(\mathrm{~m}, 1 \mathrm{H}), 1.85-1.81(\mathrm{~m}, 2 \mathrm{H})$, $1.73-1.63(\mathrm{~m}, 5 \mathrm{H}), 1.42(\mathrm{~m}, 1 \mathrm{H}), 1.23-1.09(\mathrm{~m}, 4 \mathrm{H})$, 0.91-0.79 (m, 2H); ${ }^{13} \mathrm{C} \quad \mathrm{NMR} \quad\left(75 \mathrm{MHz}, \mathrm{CDCl}_{3}\right) \quad \delta$ $174.0,166.5,150.6,143.4,139.5,138.1$ (q, $J=35.0 \mathrm{~Hz}$ ), $133.5,124.2(\mathrm{q}, J=277.7 \mathrm{~Hz}), 123.6,120.0,74.5,64.3$, $61.3,58.7,50.8,45.5,40.2,38.5,35.6,34.9,32.4,31.5$, and 28.2; HRMS (ESI+) $\mathrm{m} / \mathrm{z} \quad[\mathrm{M}+\mathrm{H}]^{+}$calcd for $\mathrm{C}_{22} \mathrm{H}_{24} \mathrm{~F}_{3} \mathrm{~N}_{4} \mathrm{OS}$ : 449.1617; found 449.1613 (error $1.0 \mathrm{ppm}$ ).

\section{MIC determination}

The test compound MICs against $M t b \mathrm{H} 37 \mathrm{Rv}$ were assessed by the microplate alamar blue assay protocol using macozinone as positive control. Compound stock solutions were prepared in DMSO at a concentration of $32 \mu \mathrm{g} / \mathrm{mL}$, and the final test concentrations ranged from 3.2 to $0.002 \mu \mathrm{g} / \mathrm{mL}$. Twofold dilutions of compounds were prepared in 7H9ADC-TG in a volume of $100 \mu \mathrm{L}$ in 96-well microplates (BD OptiluxTM, 96-well Microplates, black/clear flat bottom). The TB cultures $\left(100 \mu \mathrm{L}\right.$ inoculua of $\left.2 \times 10^{5} \mathrm{cfu} / \mathrm{mL}\right)$ were added to the media, yielding a final testing volume of $200 \mu \mathrm{L}$. The plates were incubated at $37{ }^{\circ} \mathrm{C}$. On the seventh day of incubation, $12.5 \mu \mathrm{L}$ of $20 \%$ Tween 80 , and $20 \mu \mathrm{L}$ of alamar blue (Invitrogen BioSource ${ }^{\mathrm{TM}}$ ) were added to the wells of test plate. After incubation at $37^{\circ} \mathrm{C}$ for $16-24 \mathrm{~h}$, fluorescence of the wells was measured at $530 \mathrm{~nm}$ (excitation) and $590 \mathrm{~nm}$ (emission). The MICs are defined as the lowest concentration effecting a reduction in fluorescence of $\geq 90 \%$ relative to the mean of replicate bacteria-only controls.

\section{Microsomal stability}

The selected compounds were dissolved in DMSO to make $10 \mathrm{mM}$ of stock solution. Midazolam, Dextromethorphan, Diclofenac, Omeprazole, and Phenacetin are used as controls. The $5 \mu \mathrm{L}$ of $10 \mathrm{mM}$ stock solution of the target compounds was diluted to $100 \mu \mathrm{M}$ of compound solution by adding $495 \mu \mathrm{L}$ of DMSO. The $2.5 \mu \mathrm{L}$ control/test compound was mixed with $197.5 \mu \mathrm{L}$ liver microsome (mouse and 
human) gently and preincubate at $37^{\circ} \mathrm{C}$ for $5 \mathrm{~min}$. The reaction was initiated by adding $50 \mu \mathrm{L}$ buffer/NADPH working solution $(5 \mathrm{mM})$ and mixed well. At each time point of $0 \mathrm{~min}$ and $30 \mathrm{~min}, 30 \mu \mathrm{L}$ of reaction solution was taken out and quenched by adding $300 \mu \mathrm{L}$ of internal standard $(10 \mathrm{ng} /$ $\mathrm{mL}$ ). The mixture was centrifuged at $4000 \mathrm{rpm}$ at $4{ }^{\circ} \mathrm{C}$ for 15 min. $100 \mu \mathrm{L}$ of the supernatant was mixed with $100 \mu \mathrm{L}$ distilled water and then analyzed by LC-MS/MS.

LC-MS/MS analysis was performed on a Kinetex C18 $100 \mathrm{~A}$ column $(3.0 \mathrm{~mm} \times 30 \mathrm{~mm}, 2.6 \mu \mathrm{m})$ with a gradient mobile phase of acetonitrile/water containing $0.1 \%$ formic acid at $0.8 \mathrm{~mL} / \mathrm{min}$ flow rate. LC condition: solvent $\mathrm{A}=\mathrm{H} 2 \mathrm{O}(0.1 \%$ formic acid $)$, solvent $\mathrm{B}=$ acetonitrile $(0.1 \%$ formic acid). Gradient elution: $0.00 \mathrm{~min}, 95.0 \% \mathrm{~A} ; 0.50 \mathrm{~min}$, $95 \% \mathrm{~A} ; 0.80 \mathrm{~min}, 5 \% \mathrm{~A} ; 1.50 \mathrm{~min}, 5 \% \mathrm{~A} ; 1.51 \mathrm{~min}, 95 \% \mathrm{~A}$; $2.00 \mathrm{~min}, 95 \% \mathrm{~A}$. The injection volume was $5 \mu \mathrm{L}$.

\section{In vivo pharmacokinetic profiles}

Animal Care and Welfare Committee of Institute of Materia Medica, Chinese Academy of Medical Sciences approved all animal protocols (1 Xian nong tan Street, Xicheng District, Beijing, China; protocol \#SYXK 2014-0023). All animal programs were in compliance with the Guide for the Care and Use of Laboratory Animals issued by Beijing Association on Laboratory Animal Care (BALAC). SPF male ICR mice weighing 22-23 g were divided into two groups with three mice each: one for oral administration and intravenous injection, separately. The tested compound was formulated at a concentration of $1.0 \mathrm{mg} / \mathrm{mL}$ for a dose of $10 \mathrm{mg} / \mathrm{kg}$ given orally (p.o.) and at $0.4 \mathrm{mg} / \mathrm{mL}$ for a dose of $2 \mathrm{mg} / \mathrm{kg}$ given i.v. The tested compound was formulated by $0.5 \%$ carboxymethyl cellulose and $0.5 \%$ Tween 80 for p.o. administration and with $20 \% \mathrm{HP}-\beta-\mathrm{CD}$ with $4 \mathrm{~mol} / \mathrm{L} \mathrm{HCl}$ for i.v. administration, respectively. Plasma samples were extracted with acetonitrile containing Terfenadine as an internal standard using a 20:1 extractant-to-plasma ratio. Analyte quantitation was performed by a LC/TSQ Quantum Access mass spectrometer (AB Sciex 5500). Chromatographic separation was performed on a Kinetex C18 $100 \mathrm{~A}$ column $(30 \mathrm{~mm} \times 3 \mathrm{~mm}, 2.6 \mu \mathrm{m})$ with an isocratic mobile phase of acetonitrile/water (80:20, v/v) containing $0.1 \%$ formic acid at $0.8 \mathrm{~mL} / \mathrm{min}$ flow rate. The pharmacokinetic parameters were calculated using WinNonlin software version 6.3 based on non-compartmental analysis (Pharsight Corporation, Mountain View, USA). The oral bioavailability was calculated as the ratio between the area under the curve (AUC) following intravenous administration corrected for dose $(F=($ AUCp.o. $\times$ dosei.v. $) /($ AUCi.v.×dosep.o. $))$.

Acknowledgements This work was financially supported by a grant from the CAMS Innovation Fund for Medical Sciences (CAMS-2017I2M-1-011).
Author contributions The manuscript was written through contributions of all authors. All authors have given approval to the final version of the manuscript.

\section{Compliance with ethical standards}

Conflict of interest The authors declare that they have no conflict of interest.

Publisher's note Springer Nature remains neutral with regard to jurisdictional claims in published maps and institutional affiliations.

\section{References}

1. Global Tuberculosis Report 2020. Geneva: World Health Organization; 2020.

2. Stehr M, Elamin AA, Singh M. Filling the pipeline-new drugs for an old disease. Current Topics Med Chem. 2014;14:110-29.

3. Makarov V, Manina G, Mikusova K, Möllmann U, Ryabova O, Saint-Joanis B, et al. Benzothiazinones kill Mycobacterium tuberculosis by blocking arabinan synthesis. Science. 2009;324:801-4. https://doi.org/10.1126/science.1171583.

4. Manina G, Pasca MR, Buroni S, De Rossi E, Riccardi G. Decaprenylphosphoryl- $\beta$-D-ribose 2'-epimerase from Mycobacterium tuberculosis is a magic drug target. Current Med Chem. 2010;17:3099-108. https://doi.org/10.2174/092986710791959693.

5. Mikusová K, Makarov V, Neres J. DprE1-from the discovery to the promising tuberculosis drug target. Current Pharm Design. 2014;20:4379-403. https://doi.org/10.2174/ 138161282027140630122724.

6. Makarov V, Lechartier B, Zhang M, Neres J, van der Sar AM, Raadsen SA, et al. Towards a new combination therapy for tuberculosis with next generation benzothiazinones. EMBO Mol Med. 2014;6:372-83.

7. Wolucka BA. Biosynthesis of D-arabinose in mycobacteria - a novel bacterial pathway with implications for antimycobacterial therapy. The FEBS J. 2008;275:2691-711. https://doi.org/10. 1111/j.1742-4658.2008.06395.x.

8. Mikusová K, Huang H, Yagi T, Holsters M, Vereecke D, D'Haeze W, et al. Decaprenylphosphoryl arabinofuranose, the donor of the D-arabinofuranosyl residues of mycobacterial arabinan, is formed via a two-step epimerization of decaprenylphosphoryl ribose. $\mathrm{J}$ Bacteriol. 2005;187:8020-5. https://doi.org/10.1128/jb.187.23. 8020-8025.2005.

9. Lechartier B, Hartkoorn RC, Cole ST. In vitro combination studies of benzothiazinone lead compound BTZ043 against Mycobacterium tuberculosis. Antimicrobial Agents Chemother. 2012;56:5790-3. https://doi.org/10.1128/aac.01476-12.

10. Trefzer C, Rengifo-Gonzalez M, Hinner MJ, Schneider $P$, Makarov V, Cole ST, et al. Benzothiazinones: prodrugs that covalently modify the decaprenylphosphoryl- $\beta$-D-ribose 2 -epimerase DprE1 of Mycobacterium tuberculosis. J Am Chem Soc. 2010;132:13663-5.

11. Sommer R, Neres J, Piton J, Dhar N, van der Sar A, Mukherjee R, et al. Fluorescent benzothiazinone analogues efficiently and selectively label Dpre1 in mycobacteria and actinobacteria. ACS Chem Biol. 2018;13:3184-92. https://doi.org/10.1021/acschembio. 8 b00790.

12. Makarov V, Neres J, Hartkoorn RC, Ryabova OB, Kazakova E, Šarkan M, et al. The 8-pyrrole-benzothiazinones are noncovalent inhibitors of DprE1 from Mycobacterium tuberculosis. Antimicrobial Agents Chemother. 2015;59:4446-52.

13. Zhang G, Aldrich CC. Macozinone: revised synthesis and crystal structure of a promising new drug for treating drug-sensitive and 
drug-resistant tuberculosis. Acta Crystallographica Section C. 2019;75:1031-5.

14. Richter A, Rudolph I, Möllmann U, Voigt K, Chung CW, Singh OMP, et al. Novel insight into the reaction of nitro, nitroso and hydroxylamino benzothiazinones and of benzoxacinones with Mycobacterium tuberculosis DprE1. Scientific Rep. 2018;8:13473. https://doi.org/10.1038/s41598-018-31316-6.

15. Gao C, Peng C, Shi Y, You X, Ran K, Xiong L, et al. Benzothiazinethione is a potent preclinical candidate for the treatment of drug-resistant tuberculosis. Scientific Rep. 2016;6:1-9.

16. Gao C, Ye T-H, Wang N-Y, Zeng X-X, Zhang L-D, Xiong Y, et al. Synthesis and structure-activity relationships evaluation of benzothiazinone derivatives as potential anti-tubercular agents. Bioorganic Med Chem Lett. 2013;23:4919-22.

17. Tiwari R, Miller PA, Chiarelli LR, Mori G, Šarkan M, Centárová I, et al. Design, syntheses, and anti-TB activity of 1, 3benzothiazinone azide and click chemistry products inspired by BTZ043. ACS Med Chem Lett. 2016;7:266-70.

18. Li P, Wang B, Zhang X, Batt SM, Besra GS, Zhang T, et al. Identification of novel benzothiopyranone compounds against Mycobacterium tuberculosis through scaffold morphing from benzothiazinones. Eur J Med Chem. 2018;160:157-70.

19. Karoli T, Becker B, Zuegg J, Möllmann U, Ramu S, Huang JX, et al. Identification of antitubercular benzothiazinone compounds by ligand-based design. J Med Chem. 2012;55:7940-4.

20. Lv K, Tao Z, Liu Q, Yang L, Wang B, Wu S, et al. Design, synthesis and antitubercular evaluation of benzothiazinones containing a piperidine moiety. Eur J Med Chem. 2018;151:1-8.

21. Lv K, Wang A, Tao Z, Fu L, Liu H, Wang B, et al. hERG optimizations of IMB1603, discovery of alternative benzothiazinones as new antitubercular agents. Eur J Med Chem. 2019;179:208-17.

22. Lv K, You X, Wang B, Wei Z, Chai Y, Wang B, et al. Identification of better pharmacokinetic benzothiazinone derivatives as new antitubercular agents. ACS Med Chem Lett. 2017;8:636-41.

23. Peng C-T, Gao C, Wang N-Y, You X-Y, Zhang L-D, Zhu Y-X, et al. Synthesis and antitubercular evaluation of 4-carbonyl piperazine substituted 1, 3-benzothiazin-4-one derivatives. Bioorganic Med Chem Lett. 2015;25:1373-6.

24. Piton J, Vocat A, Lupien A, Foo CS, Riabova O, Makarov V et al. Structure-based drug design and characterization of sulfonylpiperazine benzothiazinone inhibitors of DprE1 from Mycobacterium tuberculosis. Antimicrobial Agents Chemother. 2018;62.

25. Tiwari R, Miller PA, Cho S, Franzblau SG, Miller MJ. Syntheses and antituberculosis activity of 1,3-benzothiazinone sulfoxide and sulfone derived from BTZ043. ACS Med Chem Letters. 2015;6:128-33.

26. Wang A, Lv K, Tao Z, Gu J, Fu L, Liu M, et al. Identification of benzothiazinones containing an oxime functional moiety as new anti-tuberculosis agents. Eur J Med Chem. 2019;181:111595.

27. Xiong L, Gao C, Shi Y-J, Tao X, Peng C-T, Rong J et al. Metabolism of SKLB-TB1001, a potent antituberculosis agent, in animals. Antimicrobial Agents Chemother. 2018;62.

28. Xiong L, Gao C, Shi Y-J, Tao X, Rong J, Liu K-L, et al. Identification of a new series of benzothiazinone derivatives with excellent antitubercular activity and improved pharmacokinetic profiles. RSC Adv. 2018;8:11163-76.

29. Zhang R, Lv K, Wang B, Li L, Wang B, Liu M, et al. Design, synthesis and antitubercular evaluation of benzothiazinones containing an oximido or amino nitrogen heterocycle moiety. RSC Adv. 2017;7:1480-3.
30. Zhang G, Howe M, Aldrich CC. Spirocyclic and bicyclic 8nitrobenzothiazinones for tuberculosis with improved physicochemical and pharmacokinetic properties. ACS Med Chem Lett. 2019;10:348-51.

31. Shaikh MH, Subhedar DD, Arkile M, Khedkar VM, Jadhav N, Sarkar D, et al. Synthesis and bioactivity of novel triazole incorporated benzothiazinone derivatives as antitubercular and antioxidant agent. Bioorg Med Chem Lett. 2016;26:561-9. https://doi. org/10.1016/j.bmcl.2015.11.071.

32. Majewski MW, Tiwari R, Miller PA, Cho S, Franzblau SG, Miller MJDesign. syntheses, and anti-tuberculosis activities of conjugates of piperazino-1,3-benzothiazin-4-ones (pBTZs) with 2,7-dimethylimidazo [1,2-a]pyridine-3-carboxylic acids and 7-phenylacetyl cephalosporins. Bioorg Med Chem Lett. 2016;26:2068-71. https://doi.org/10.1016/j.bmcl.2016.02.076.

33. Armenise D, Muraglia M, Florio MA, De Laurentis N, Rosato A, Carrieri A, et al. 4H-1,4-benzothiazine, dihydro-1,4-benzothiazinones and 2-amino-5-fluorobenzenethiol derivatives: design, synthesis and in vitro antimicrobial screening. Archiv der Pharmazie. 2012;345:407-16. https://doi.org/10.1002/ardp. 201100309.

34. Tiwari R, Moraski GC, Krchňák V, Miller PA, Colon-Martinez $\mathrm{M}$, Herrero $\mathrm{E}$, et al. Thiolates chemically induce redox activation of BTZ043 and related potent nitroaromatic anti-tuberculosis agents. J Am Chem Soc. 2013;135:3539-49.

35. Foo CS, Lechartier B, Kolly GS, Boy-Röttger S, Neres J, Rybniker $\mathrm{J}$, et al. Characterization of DprE1-mediated Benzothiazinone resistance in mycobacterium tuberculosis. Antimicrobial Agents Chemother. 2016;60:6451-9. https://doi.org/10.1128/aac.01523-16.

36. Trefzer C, Škovierová H, Buroni S, Bobovská A, Nenci S, Molteni $\mathrm{E}$, et al. Benzothiazinones are suicide inhibitors of mycobacterial decaprenylphosphoryl- $\beta$-D-ribofuranose 2'-oxidase DprE1. J Am Chem Soc. 2012;134:912-5. https://doi.org/10. 1021/ja211042r.

37. Neres J, Pojer F, Molteni E, Chiarelli LR, Dhar N, Boy-Röttger S, et al. Structural basis for benzothiazinone-mediated killing of Mycobacterium tuberculosis. Sci Transl Med. 2012;4:150ra21. https://doi.org/10.1126/scitranslmed.3004395.

38. Fleming FF, Yao L, Ravikumar PC, Funk L, Shook BC. Nitrilecontaining Pharmaceuticals: efficacious roles of the nitrile pharmacophore. J Med Chem. 2010;53:7902-17. https://doi.org/10. 1021/jm100762r.

39. Liu L, Kong C, Fumagalli M, Savková K, Xu Y, Huszár S, et al. Design, synthesis and evaluation of covalent inhibitors of DprE1 as antitubercular agents. Eur J Med Chem. 2020;208:112773. https://doi.org/10.1016/j.ejmech.2020.112773.

40. Richter A. Synthese von benzothiazinonen und derivaten als DprE1 hemmstoffe mit antimykobakterieller Aktivität. MartinLuther-Universität Halle-Wittenberg: Germany, 2017.

41. Zhang G, Aldrich CC. Macozinone: revised synthesis and crystal structure of a promising new drug for treating drug-sensitive and drug-resistant tuberculosis. Acta crystallographica Section C. Struct Chem. 2019;75:1031-5. https://doi.org/10.1107/ s2053229619009185.

42. Makarov AV, Cole ST, Möllmann U. New benzothiazinone derivatives and their use as antibacterial agents. Google Patents, 2011.

43. Leeson PD, Springthorpe B. The influence of drug-like concepts on decision-making in medicinal chemistry. Nat Rev Drug Discov. 2007;6:881-90. 